\title{
Dissociation between muscle metabolism and oxygen kinetics during recovery from exercise in patients with chronic heart failure
}

\author{
A Hanada, K Okita, K Yonezawa, M Ohtsubo, T Kohya, T Murakami, H Nishijima, \\ M Tamura, A Kitabatake
}

\begin{abstract}
Objective-To estimate muscle metabolism and oxygen delivery to skeletal muscle in patients with chronic heart failure.

Methods-13 patients with chronic heart failure and 15 controls performed calf plantar flexion for six minutes at a constant workload of $50 \%$ of one repetition maximum. During recovery from exercise, skeletal muscle content of oxygenated haemoglobin (oxy-Hb) and the level of phosphocreatine (PCr) were measured by near-infrared spectroscopy and ${ }^{31} \mathrm{P}$-magnetic resonance spectroscopy, respectively.

Results-The mean (SD) time constants of $\mathrm{PCr}$ and oxy-Hb during recovery from exercise were significantly greater in patients with chronic heart failure than in normal subjects $(\tau \mathrm{PCr}: 76.3$ (30.2) s v 36.5 (5.8) s; $\tau$ oxy-Hb: 48.3 (7.3) s v 30.1 (7.7) s; p < 0.01). Both time constants were similar in normal subjects, while the $\tau$ PCr was significantly greater than the $\tau$ oxy-Hb in patients with chronic heart failure.

Conclusions-The slower recovery of PCr compared with oxy-Hb in patients with chronic heart failure indicates that haemoglobin resaturation is not a major rate limiting factor of $\mathrm{PCr}$ resynthesis. It is suggested that muscle metabolic recovery may depend more on oxygen utilisation than on haemoglobin resaturation or oxygen delivery in patients with chronic heart failure. (Heart 2000;83:161-166)
\end{abstract}

Keywords: near-infrared spectroscopy; ${ }^{31} \mathrm{P}$-magnetic resonance spectroscopy; chronic heart failure; exercise tolerance

Studies have shown that the degree of exercise intolerance in patients with chronic heart failure is not significantly correlated with the extent of the central haemodynamic disturbance. ${ }^{12}$ This means that exercise capacity is not limited only by haemodynamics but also by peripheral abnormality. Studies using phosphorus-31 nuclear magnetic resonance spectroscopy $\left({ }^{31} \mathrm{P}-\mathrm{MRS}\right)$ have shown that peripheral muscle metabolic abnormalities during exercise are important contributors to exercise intolerance in patients with chronic heart failure..$^{3-8}$ Recent studies using nearinfrared spectroscopy (NIRS) to evaluate skeletal muscle oxygen kinetics have shown that peripheral muscle oxygenation is impaired during systemic exercise in patients with chronic heart failure. ${ }^{910}$ Both muscle metabolism and muscle oxygen kinetics are important determinants of exercise capacity and these factors have been separately evaluated in patients with chronic heart failure. In normal subjects, only a few studies have assessed both muscle metabolism and oxygen kinetics. McCully et al simultaneously measured oxygenated haemoglobin (oxy-Hb) and phosphocreatine (PCr) recovery after submaximal exercise in normal subjects using NIRS and ${ }^{31} \mathrm{P}-\mathrm{MRS}$ respectively, and found that the time constants of these indices were similar. ${ }^{11}$ They suggested that oxy- $\mathrm{Hb}$ recovery is rate limiting for ATP synthesis, evaluated as the rate of PCr recovery after submaximal exercise. In patients with chronic heart failure, both skeletal muscle metabolism and oxygen delivery are impaired and these abnormalities are potential contributors to exercise intolerance. Therefore the combination of ${ }^{31} \mathrm{P}-\mathrm{MRS}$ and NIRS would appear to be useful for assessing exercise intolerance. However, these methods have not been used in combination in patients with chronic heart failure during exercise.

To elucidate the relation between muscle metabolism and oxygen kinetics, we measured $\mathrm{PCr}$ and oxy-Hb during recovery from submaximal constant load exercise in patients with chronic heart failure and in normal subjects. We also investigated the relations between these factors and systemic exercise capacity.

\section{Methods}

SUBJECTS

We studied 15 patients with chronic heart failure, mean (SD) age 58 (8) years, and 16 age matched normal subjects. The mean ejection fraction of the patients with chronic heart failure measured by radioisotope scintigraphy was 29 (13)\%. Eight had symptoms consistent with New York Heart Association class II and seven with class III. Heart failure was attributed to idiopathic dilated cardiomyopathy in all patients. At the time of the study all patients were receiving diuretics, seven were receiving $\beta$ blockers, and 11 were receiving angiotensin converting enzyme inhibiting agents. Six patients were taking digitalis. Patients with peripheral vascular disease were excluded from the study. The normal subjects were sedentary 
healthy volunteers, mean age 49 (11) years, with no known medical problems. Written informed consent was obtained from each subject.

SYSTEMIC EXERCISE

Upright bicycle exercise was performed with a ramp protocol $(15 \mathrm{~W} / \mathrm{min}$ after a three minute warm up at $0 \mathrm{~W}$ ) using an electromechanical bicycle ergometer (Corvial 400, Lode, Groningen, Holland). During exercise, respiratory gas analysis was performed by a breath by breath apparatus (Aeromonitor AE-280, Minato Medical Science, Osaka, Japan). Peak oxygen uptake (peak $\dot{\mathrm{V}}_{2}$ ) and the ventilatory anaerobic threshold were determined by the V slope method described by Beaver and Wasserman. ${ }^{12}$

\section{LOCAL EXERCISE}

Subjects performed supine plantar flexion of the right calf muscle. Their right foot was fixed on a pedal shaped lever attached to a basket in which appropriate weights were placed. The subjects were immobilised on the platform by a system of Velcro straps at the knee, ankle, chest, and shoulders. Before the study, muscle strength was measured in all subjects by the one repetition maximum (1 RM) method, which measures the maximum weight that can be lifted only once.

Using magnetic resonance imaging, the maximum calf flexor muscle cross sectional area (MCA) was determined. Conventionally there have been two ways to decide the workload in skeletal muscle exercise, as a function of MCA and MVC (maximal voluntary contraction). We used $1 \mathrm{RM}$ as MVC and the workload was adjusted to $50 \%$ of $1 \mathrm{RM}$. Plantar flexion was performed once every 1.5 seconds for six minutes against a pedal. Measurements were obtained from the one minute rest period before exercise through the six minute recovery period after exercise.

\section{PHOSPHORUS-31 nuclear magnetic resonance spectroscopy}

We obtained ${ }^{31} \mathrm{P}$-MRS measurements using an $80 \mathrm{~mm}$ surface coil in a $55 \mathrm{~cm}$ bore, 1.5 Tesla superconducting magnet (Magnetom H15, Siemens, Erlangen, Germany). Shimming was adjusted using a proton signal from water. Spectra were obtained with a pulse width of $500 \mathrm{~ms}$, a transmitter voltage of $20 \mathrm{~V}$, and a repetition time of $1000 \mathrm{~ms}$, and four scans were performed and averaged for each spectrum. $\mathrm{PCr}$ is expressed as $[\mathrm{PCr}] /([\mathrm{PCr}]+[\mathrm{Pi}])$, where $\mathrm{Pi}$ is inorganic phosphate. The degree of $\mathrm{PCr}$ change (PCr depletion) was calculated as: $\mathrm{PCr}$ depletion $=($ rest $\mathrm{PCr}-$ peak $\mathrm{PCr}) /$ rest $\mathrm{PCr}$.

The muscle $\mathrm{pH}$ was calculated from the changes in the chemical shifts of Pi relative to $\mathrm{PCr}$ as previously described. ${ }^{13}$ As previous studies have shown the appropriateness of using monoexponential fitting to describe the rate of PCr recovery, ${ }^{14}{ }^{15}$ we estimated it using time constants. $\mathrm{PCr}$ recovery after exercise was fitted to a single exponential curve obtained by least squares regression, and the time constant for PCr recovery ( $\tau \mathrm{PCr}$ ) was calculated as follows: $[\mathrm{PCr}]=\mathrm{C} 1+\mathrm{C} 2\left(1-\mathrm{e}^{-\mathrm{kt}}\right)$, where $[\mathrm{PCr}]$ is the PCr concentration, $\mathrm{C} 1$ is the initial $[\mathrm{PCr}$, $\mathrm{C} 2$ is the difference between the final and initial [PCr], $\mathrm{t}$ is time, and $\mathrm{k}$ is the rate constant $(1 / \mathrm{k}=\tau)$.

NEAR-INFRARED SPECTROSCOPY

NIRS was performed with a dual wave spectrometer (HEO100, Omron, Tokyo, Japan), a tissue oximeter that uses a two wavelength light emitting diode (LED), with wavelengths of 760 and $840 \mathrm{~nm}$, as a light source. The basic principles of $\mathrm{NIRS}^{16}$ and in vitro results ${ }^{17}$ obtained with this tissue oximeter have been described previously. The NIRS probe, which has a photodiode in the centre and a near-infrared LED on each side, was attached to the medial portion of the calf muscle and fixed with a rubber strap to prevent displacement during exercise. All studies were performed using the same auto gain settings on the spectrometer. Subjects rested during the one minute gain setting. After exercise begins, oxy-Hb saturation is depleted from the stable baseline and then reaches a plateau indicating the balance of oxygen demand and supply in muscle tissue. After exercise is completed, the oxy-Hb saturation increases until it reaches a plateau. Data sampled every 0.5 seconds were fed into a personal computer and saved as a file. As in previous studies discussing recovery rate of oxy- $\mathrm{Hb},{ }^{11}{ }^{18}$ we evaluated the recovery kinetics by means of time constants. The oxy-Hb recovery was fitted to a single exponential curve and the time constant for oxy- $\mathrm{Hb}$ recovery $(\tau$ oxy-Hb) was calculated as the $\tau$ PCr. Measurements by ${ }^{31} \mathrm{P}-\mathrm{MRS}$ and NIRS were obtained with the same protocol on alternate days within a week.

\section{REPRODUCIBILITY}

We have already reported the reproducibility of MRS data during local muscle exercise. ${ }^{19}$ Furthermore, we confirmed the reproducibility of $\mathrm{PCr}$ recovery with the same protocol in seven patients with chronic heart failure (coefficient of variation $8 \%$ ). Walter et al also reported the good reproducibility of $\tau$ of $\mathrm{PCr}$ recovery. ${ }^{20}$ To investigate the reproducibility of the NIRS data, five subjects underwent two tests within a one week period. The coefficient of variation between the two tests was $14 \%$, which was small enough for the present study.

\section{STATISTICAL ANALYSIS}

All data were entered into a database on a personal computer and analysed with a commercial statistical package (Stat View-J 4.21, Abacus Concepts Inc and Igor, Wave Metrics). Significant differences between group means were analysed by a non-paired $t$ test. Differences among the $\tau$ PCr and the $\tau$ oxy-Hb were analysed by analysis of variance for within and between patients with chronic heart failure and normal subjects. A level of $\mathrm{p}<0.05$ was considered statistically significant. Data are expressed as means (SD). 
Table 1 Haemodynamic and respiratory gas responses to maximal bicycle exercise

\begin{tabular}{lcc}
\hline & $\begin{array}{l}\text { Normal subjects } \\
(n=15)\end{array}$ & $\begin{array}{l}\text { Patients with } \\
C H F(n=13)\end{array}$ \\
\hline Peak heart rate (beats/min) & $169(15)$ & $146(23)^{\star}$ \\
Peak $\dot{V}_{2}(\mathrm{ml} / \mathrm{min} / \mathrm{kg})$ & $30.0(6.7)$ & $19.2(2.7)^{\star}$ \\
AT $(\mathrm{ml} / \mathrm{min} / \mathrm{kg})$ & $18.8(3.9)$ & $12.8(1.8)^{\star}$ \\
Peak workload (W) & $185 \quad(53)$ & $106 \quad(15)^{\star}$ \\
\hline
\end{tabular}

Values are means (SD).

${ }^{\star} \mathrm{p}<0.01 v$ normal subjects.

AT, anaerobic threshold; CHF, chronic heart failure; $\dot{V}_{2}$, oxygen consumption.

Table 2 Indices of local exercise

\begin{tabular}{lcc}
\hline & $\begin{array}{l}\text { Normal subjects } \\
(n=15)\end{array}$ & $\begin{array}{l}\text { Patients with } \\
C H F(n=13)\end{array}$ \\
\hline 1 RM (kg) & $43.5(9.6)$ & $35.9(10.7)$ \\
MCA (cm $\left.{ }^{2}\right)$ & $52.9(9.2)$ & $45.5(5.9)^{\star}$ \\
1 RM/MCA & $0.78(0.34)$ & $0.82(0.18)$ \\
Muscle PCr & & \\
$\quad$ Rest & $0.95(0.02)$ & $0.91(0.05)$ \\
$\quad$ Peak & $0.48(0.06)$ & $0.40(0.01)^{\star}$ \\
PCr depletion & $0.50(0.06)$ & $0.56(0.09)^{\star}$ \\
$\quad \tau$ PCr (s) & $36.5(5.8)$ & $76.3(30.2)^{\star}$ \\
Muscle pH & & \\
$\quad$ Rest & $7.00(0.10)$ & $7.05(0.08)$ \\
Peak & $6.75(0.03)$ & $6.56(0.01)^{\star}$ \\
$\tau$ Oxy-Hb (s) & $30.1(7.7)$ & $48.3(7.3)^{\star}$ \\
\hline
\end{tabular}

Values are means (SD).

${ }^{\star} \mathrm{p}<0.05$ v normal subjects.

$\mathrm{CHF}$, chronic heart failure; MCA, maximum muscle cross sectional area; oxy- $\mathrm{Hb}$, oxyhaemoglobin; $\mathrm{PCr}$, phosphocreatine; 1 $\mathrm{RM}$, one repetition maximum method.

\section{Results}

SYSTEMIC EXERCISE

The peak heart rate, peak $\dot{\mathrm{V}}_{2}$, and anaerobic threshold were significantly greater in the normal subjects than in the patients with chronic heart failure (table 1). The maximum workload was also greater in the normal subjects than in the patients with chronic heart failure.

LOCAL EXERCISE

Three subjects (two patients and one normal subject) could not complete the six minute exercise and they were excluded from the statistical analysis. As shown in table 2,
$1 \mathrm{RM}$ tended to be greater in normal subjects than in patients with chronic heart failure but there was no statistical difference. The MCA was significantly greater in normal subjects. The $1 \mathrm{RM} / \mathrm{MCA}$ was similar in both groups.

\section{${ }^{31}$ P-MRS MEASUREMENTS}

The resting muscle $\mathrm{PCr}$ and $\mathrm{pH}$ value were similar in both groups (table 2). Peak muscle $\mathrm{PCr}$ and peak $\mathrm{pH}$ were significantly lower in patients with chronic heart failure than in normal subjects. Representative data of ${ }^{31} \mathrm{P}$ MRS and NIRS spectra after exercise in a patient and a normal subject are shown in fig 1 . The rate of PCr recovery, evaluated as the $\tau$ PCr, was significantly greater in patients with chronic heart failure than in normal subjects (table 2), indicating that $\mathrm{PCr}$ recovery was impaired in patients with chronic heart failure.

In fig 2, normal subjects showed a significantly smaller value of the $\tau$ PCr and greater anaerobic threshold, while patients with chronic heart failure showed greater $\tau \mathrm{PCr}$ and lower anaerobic threshold (left panel). The $\tau$ PCr was significantly correlated with anaerobic threshold when both groups were included $(R=0.54, \mathrm{p}<0.01)$.

\section{NIRS MEASUREMENTS}

The rate of oxy-Hb recovery evaluated as the $\tau$ oxy-Hb was significantly greater in patients with chronic heart failure than in normal subjects (table 2). As well as the $\tau$ PCr, normal subjects showed significantly smaller $\tau$ oxy- $\mathrm{Hb}$ and greater anaerobic threshold, while patients with chronic heart failure showed greater $\tau$ oxy-Hb and lower anaerobic threshold (fig 2, right panel). The $\tau$ oxy-Hb was correlated with the anaerobic threshold when both groups were included $(R=0.70, \mathrm{p}<0.01)$.
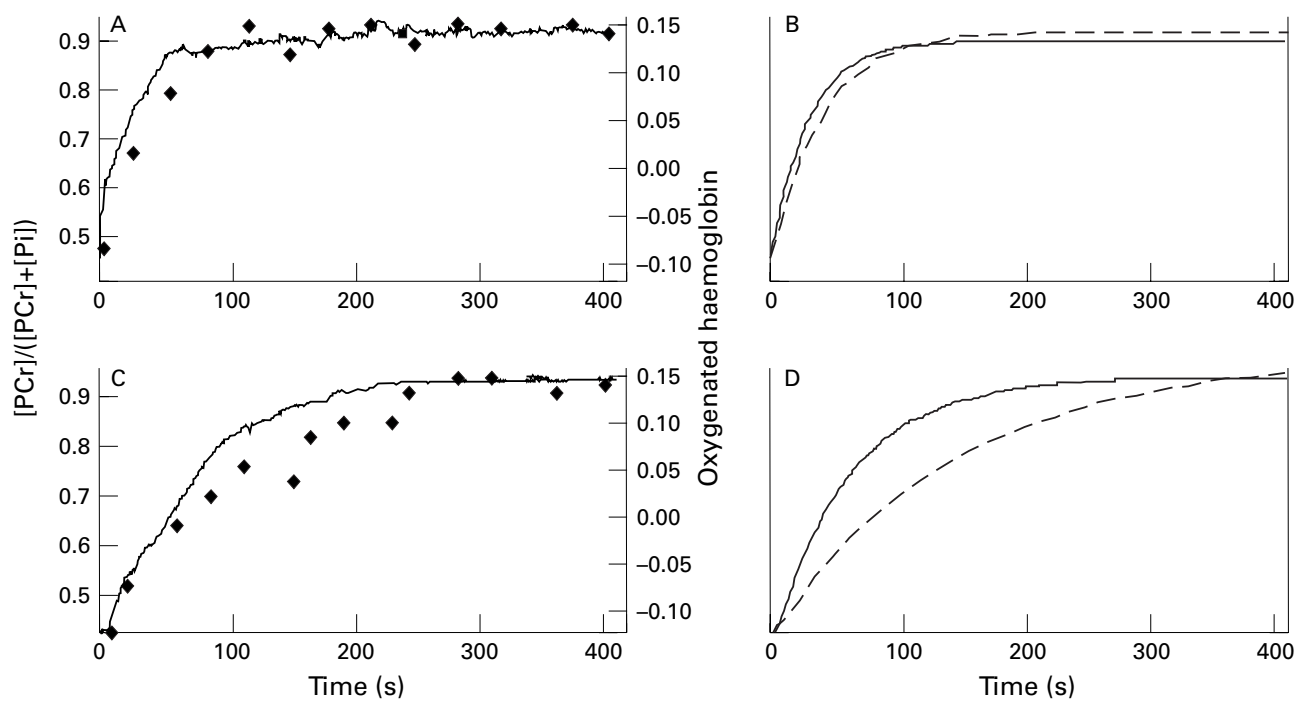

Figure 1 Representative spectra showing recovery of phosphocreatine (PCr, filled symbols) and oxygenated haemoglobin (oxy-Hb, solid line) in normal subjects $(A)$ and patients with chronic heart failure $(C)$. Each dataset is fitted with a single exponential curve $(B$ and $D)$. A dotted line indicates the fitting curve of PCr and a solid line shows that of oxy-Hb. The time constants are as follows: $B$ (normal subject): $\tau$ oxy- $H b=28 \mathrm{~s} ; \tau \mathrm{PCr}=33 \mathrm{~s} . \mathrm{D}$ (patient with chronic heart failure): $\tau$ $o x y-H b=53 s ; \tau P C r=110$ s. $\tau$ oxy-Hb, time constant for oxy-Hb resaturation; $\tau$ PCr, time constant for PCr resynthesis. 

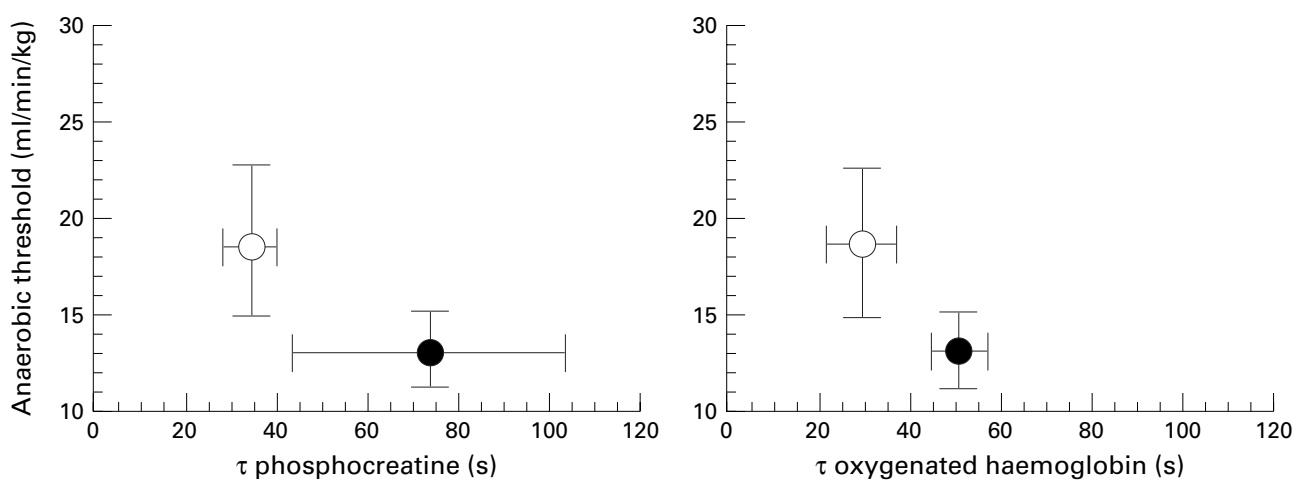

Figure 2 Relation between the time constants and the anaerobic threshold in patients with chronic heart failure (filled circles) and normal subjects (empty circles). Left panel shows correlation between anaerobic threshold and $\tau$ PCr and right panel shows anaerobic threshold and $\tau$ oxy-Hb.

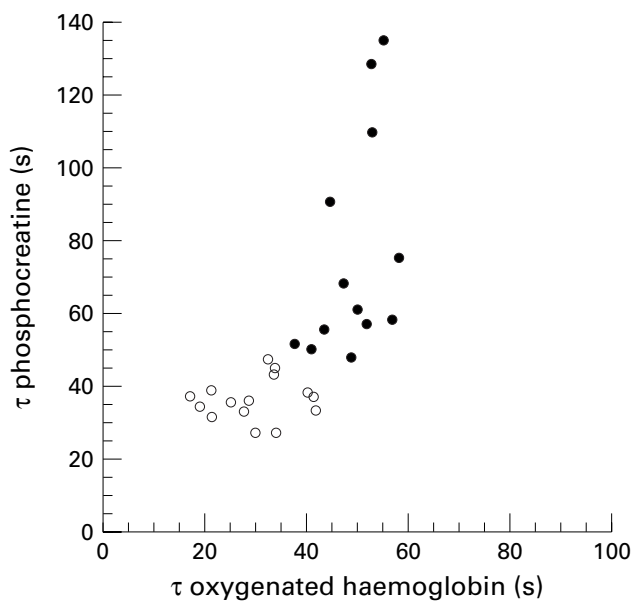

Figure 3 The relation between $\tau$ PCr and $\tau$ oxy- $\mathrm{Hb}$ presented by two dimensional plotting. Empty circles indicate normal subjects and filled circles indicate patients with chronic heart failure.

COMPARISON BETWEEN ${ }^{31}$ P-MRS AND NIRS MEASUREMENTS

These results are shown in fig 3. The $\tau$ PCr and the $\tau$ oxy-Hb was similar in normal subjects. In patients with chronic heart failure, however, the $\tau$ PCr was significantly greater than the $\tau$ oxy-Hb. Moreover, the difference in the $\tau \mathrm{PCr}$ between the two groups was remarkably greater than the difference in the $\tau$ oxy-Hb. The $\tau$ PCr in patients with chronic heart failure showed a wide variance.

\section{Discussion}

MAJOR FINDINGS

Both the $\tau$ PCr and the $\tau$ oxy-Hb after exercise were greater in patients with chronic heart failure than in normal subjects. These time constants correspond to $\mathrm{PCr}$ resynthesis and haemoglobin resaturation. ${ }^{11}$ Thus our present findings indicate that $\mathrm{PCr}$ resynthesis and haemoglobin resaturation were impaired after exercise in patients with chronic heart failure. In normal subjects, the $\tau$ oxy-Hb was similar to the $\tau$ PCr, which was consistent with previous findings. ${ }^{11}$ It suggested that the rate of haemoglobin resaturation matches the rate of oxygen utilisation after exercise to resynthesise $\mathrm{PCr}$ in skeletal muscle in normal subjects. However, the $\tau$ PCr was significantly greater than the $\tau$ oxy- $\mathrm{Hb}$ in patients with chronic heart failure in the present study, indicating that PCr resynthesis was significantly delayed compared with haemoglobin resaturation. Therefore the rate of haemoglobin resaturation does not appear to be a major determinant of muscle metabolic recovery, evaluated as $\mathrm{PCr}$ resynthesis in patients with chronic heart failure.

A possible reason for the significant delay in PCr resynthesis in muscles is impaired oxygen utilisation in muscle mitochondria or impaired oxygen diffusion to mitochondria from capillaries. In other words, muscle metabolic recovery may depend to a greater extent on the capacity of oxygen utilisation rather than on haemoglobin resaturation or oxygen delivery in patients with chronic heart failure.

${ }^{31}$ P-MRS MEASUREMENTS

During recovery period after exercise, the rate of $\mathrm{PCr}$ recovery represents the maximum oxidative capacity. ${ }^{6}{ }^{16}$ In the present study, PCr resynthesis was impaired after submaximal exercise in patients with chronic heart failure, and the rate of $\mathrm{PCr}$ resynthesis, evaluated as the $\tau \mathrm{PCr}$, may contribute to the anaerobic threshold. These findings suggest that the oxidative capacity in skeletal muscle is impaired and may affect performance during systemic exercise in patients with chronic heart failure.

Another possible reason for the greater $\tau \mathrm{PCr}$ is more intense exercise in patients with chronic heart failure. However, we corrected the workload using $1 \mathrm{RM}$. There was no significant difference in $1 \mathrm{RM}$ per MCA in normal subjects and patients with chronic heart failure, which means that the intensities of workloads per muscle area in both groups were similar (table 2). In addition, table 2 shows that the difference in the $\tau$ PCr between two groups is much greater than the difference in the PCr depletion. This suggests that the contribution of greater $\mathrm{PCr}$ depletion to the delayed $\tau$ PCr was small in patients with chronic heart failure. McCully et al also reported that low muscle $\mathrm{pH}$ (under 7.0) slowed the rate of $\mathrm{PCr}$ recovery as a result of the effect of $\mathrm{H}^{+}$concentration. ${ }^{11}$ Therefore a greater decrease in muscle $\mathrm{pH}$ may affect the delayed $\tau \mathrm{PCr}$ in patients with chronic heart failure. However, in the present study all 
patients showed a greater $\tau$ PCr than did normal subjects within the $\mathrm{pH}$ range 6.58 to 6.75 .

NIRS MEASUREMENTS

Our study showed that haemoglobin resaturation was impaired after submaximal exercise in patients with chronic heart failure. What is the meaning of this impaired haemoglobin resaturation? The rate of haemoglobin resaturation after exercise is believed to be determined by oxygenated blood flow in muscles and muscle oxygen uptake. Accelerated postexercise oxygen uptake (oxygen debt) may have been responsible for the delayed haemoglobin resaturation in patients with chronic heart failure. Belardinelli et al reported similar findings. ${ }^{10}$ Furthermore, previous studies have shown that cardiovascular stress is minimal and muscle perfusion is similar during small muscle mass exercise in patients with chronic heart failure and in normal subjects. ${ }^{1421}$ Thus the rate of haemoglobin resaturation might be determined mainly by excessive muscle oxygen uptake rather than by reduced blood flow in patients with chronic heart failure. However, if the $\tau$ oxy- $\mathrm{Hb}$ is determined by muscle oxygen uptake, the rate of haemoglobin resaturation should be consistent with the rate of $\mathrm{PCr}$ resynthesis because the major source of oxygen debt is PCr depletion. ${ }^{13}$ The present study showed a significant delay in PCr resynthesis compared with haemoglobin resaturation in patients with chronic heart failure.

One possible explanation for the delayed haemoglobin resaturation is that the resaturation may be affected by the oxygen debt but may occur earlier than repayment of the oxygen debt or PCr depletion, because of impaired oxygen utilisation of muscle mitochondria or impaired oxygen diffusion to mitochondria from capillaries. The second possible reason for the delayed haemoglobin resaturation is that it may mainly reflect an impaired oxygenated blood flow after exercise in patients with chronic heart failure. It has been shown that muscle blood flow remains normal even in patients with chronic heart failure during small muscle mass exercise. However, measurements in previous studies were performed by venous occlusion plethysmography or catheter based measurements, which evaluate non-selective blood flow. Plethysmography measures the whole limb blood flow, including the flow in skin and non-exercising muscles. A catheter is usually inserted into the femoral vein and also provides the whole limb blood flow measurement. In contrast, NIRS provides information about the flow in localised exercising muscle. It is possible that perfusion to exercising muscle is selectively impaired in patients with chronic heart failure.

\section{CLINICAL IMPLICATIONS}

Our study showed that muscle metabolism and oxygen delivery are two important factors that determine muscle function in patients with chronic heart failure. Estimating both variables with ${ }^{31} \mathrm{P}-\mathrm{MRS}$ and NIRS independently, we could acquire detailed information about the mechanism that predominantly determined the exercise capacity in each patient. We could assume that different kinds of treatment may have a differential effect on muscle metabolism or oxygen delivery, for example, muscle training, ${ }^{22}$ medication, and so on. This may help us to plan better treatments to improve their exercise intolerance and enable us to evaluate their effects appropriately.

STUDY LIMITATIONS

Although reproducibility of the $\tau$ PCr and the $\tau$ oxy-Hb was proved, we could not acquire both measurements simultaneously because of the technical limitation of the NIRS device. Second, we have included patients treated with and without $\beta$ blocker agents. This might affect the muscle metabolism. However, $\tau$ PCr and $\tau$ oxy-Hb in this study were not significantly different between patients with and without $\beta$ blocker treatment $(p=0.33$ and $p=0.38, \tau$ $\mathrm{PCr}$ and $\tau$ oxy-Hb, respectively).

CONCLUSIONS

Both PCr resynthesis and haemoglobin resaturation during recovery from exercise were impaired in patients with chronic heart failure. Remarkably, the recovery of PCr was slower than haemoglobin resaturation in patients with chronic heart failure. Furthermore the $\tau$ PCr and the $\tau$ oxy-Hb in normal subjects were similar, whereas the $\tau$ PCr in patients with chronic heart failure was significantly slower than the $\tau$ oxy-Hb. Our results suggest that haemoglobin resaturation is not the only rate limiting factor for PCr resynthesis in patients with chronic heart failure. Muscle metabolic recovery may depend on the capacity of oxygen utilisation rather than on haemoglobin resaturation or oxygen delivery in the presence of chronic heart failure. 1 Massie BM, Conway $\mathrm{M}$, Yonge $\mathrm{R}$, et al. Skeletal muscle metabolism in patients with congestive heart failure: relation to clinical

2 Massie BM, Conway M, Rajagopalan B, et al. Skeletal muscle metabolism during exercise under ischemic conditions in congestive heart failure: evidence for abnormalities unrelated to blood flow. Circulation 1988;78:320-6.

3 Wilson JR, Fink L, Maris J, et al. Evaluation of energy metabolism in skeletal muscle of patients with chronic heart failure with gated phosphorus-31 nuclear magnetic resonance. Circulation 1985;71:57-62.

4 Weiner DH, Fink LI, Maris J, et al. Evaluation of energy metabolism in skeletal muscle bioenergetics during exercise in patients with heart failure: role of reduced muscle blood flow. Circulation 1986;73:1127-36.

5 Mancini DM, Ferraro N, Tuchler M, et al. Detection of abnormal calf muscle metabolism in patients with heart failure using phosphorus-31 nuclear magnetic resonance. failure using phosphorus-31 nucle

6 Mancini DM, Reichek N, Chance B, et al. Contribution of Mancini DM, Reichek $\mathrm{N}$, Chance B, et al. Contribution of
skeletal muscle atrophy to exercise intolerance and altered muscle metabolism in heart failure. Circulation 1992;85: muscle m

7 Mancini DM, Coyle E, Coggan A, et al. Contribution of intrinsic skeletal muscle changes to ${ }^{31} \mathrm{P}$ NMR skeletal muscle metabolic abnormalities in patients with heart failure. Circulation 1989;80:1338-46.

8 Okita K, Yonezawa K, Nishijima H, et al. Skeletal muscle metabolism limits exercise capacity in patients with chronic heart failure. Circulation 1998;98:1886-91.

9 Matsui S, Tamura N, Hirakawa T, et al. Assessment of working skeletal muscle oxygenation in patients with chronic heart failure. Am Heart f 1995;129:690-5.

10 Belardinelli R, Barstow TJ, Nguyen P, et al. Skeletal muscle oxygenation and oxygen uptake kinetics following constant work rate exercise in chronic congestive heart failure. $A m \mathcal{F}$ Work rate exercise in chronic

11 McCully KK, Iotti S, Kendrick K, et al. Simultaneous in vivo measurements of $\mathrm{HbO}_{2}$ saturation and PCr kinetics vivo measurements of $\mathrm{HbO}_{2}$ saturation and PCr kinetics 10 . 
12 Beaver WL, Wasserman K. A new method for detecting anaerobic threshold by gas exchange. F Appl Physiol anaerobic thresh

13 Taylor DJ, Styles P, Matthews DA, et al. Energetics of human muscles: exercise induced ATP depletion. Magn Reson Med 1986;3:44-54.

14 Meyer RA. A linear model of muscle respiration explains monoexponential phosphocreatune changes. Am f Physiol 1988;254:C548-53

15 Di Prampero PE, Margaria R. Mechanical efficiency of phosphagen (ATP+CP) splitting and its speed of resynthesis. Pflugers Arch 1969;308:197-202.

16 Chance B, Dait TM, Chang C, et al. Recovery from exercise-induced desaturation in the quadriceps muscles of elite competitive rowers. Am 7 Physiol 1992;262:C766-75.

17 Shiga $\mathrm{T}$, Yamamoto $\mathrm{K}$, Tanabe $\mathrm{K}$, et al. Study of an algorithm based on model experiments and diffusion theory for a portable tissue oximeter. F Biomed Optics 1997; 2:154-61.
18 McCully KK, Halber C, Posner JD. Exercise-induced changes in oxygen saturation in the calf muscles of elderly subjects with peripheral vascular disease. If Gerontol 1994;49:B128-34.

19 Nishijima H, Nishida M, Anzai T, et al. A simple ergometer for ${ }^{31} \mathrm{P}$ NMR spectroscopy during dynamic forearm exercise in a whole body magnetic resonance imaging system. Fpn Heart f 1992;33:185-92.

20 Walter G, Vandenborne K, McCully KK, et al. Noninvasive measurement of phosphocreatine recovery kinetics in single human muscles. Am F Physiol 1997;272:C525-34.

21 Arnolda L, Conway M, Doleck $M$, et al. Skeletal muscle metabolism in heart failure: a en muscle Clin Sci 1990; resonance 79:583-9.

22 Ohtsubo M, Yonezawa K, Nishijima H, et al. Metabolic abnormality of calf skeletal muscle is improved by localised muscle training without changes in blood flow in chronic muscle training without changes in
heart failure. Heart 1997;78:437-43. 